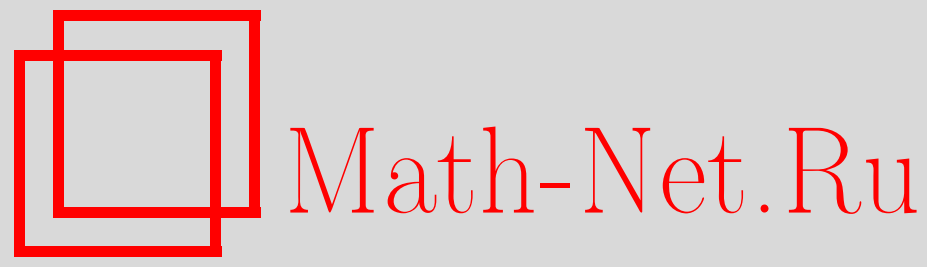

А. Н. Паршин, О голоморфных представлениях дискретных групп Гейзенберга, Функи. анализ и его прил., 2010, том 44, выпуск 2, 92-96

DOI: https://doi.org/10.4213/faa2992

Использование Общероссийского математического портала Math$\mathrm{Net.Ru}$ подразумевает, что вы прочитали и согласны с пользовательским соглашением

http://www . mathnet.ru/rus/agreement

Параметры загрузки:

IP : 18.209 .158 .208

26 апреля 2023 г., 03:46:32

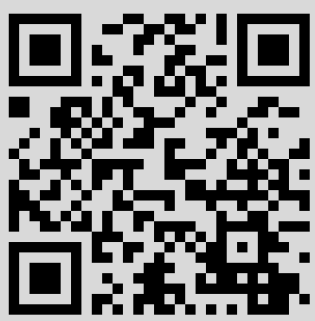


УДК 512.74

\title{
О голоморфных представлениях дискретных групп Гейзенберга*
}

\author{
(c) 2010. А. Н. ПАРшин
}

Пусть $H, H^{\prime}, C$ - конечно порожденные абелевы группы, и пусть $\langle-,-\rangle$ : $H \times H^{\prime} \rightarrow C$ - билинейное отображение. Мы называем дискретной группой Гейзенберга $G$ множество $H \times H^{\prime} \times C$ с законом композиции $(n, p, c)(m, q, a)=$ $(n+m, p+q, c+a+\langle n, q\rangle)$, где $n, m \in H, p, q \in H^{\prime}, c, a \in C$. Введем комплексные торы $\mathbb{T}_{H}=\operatorname{Hom}\left(H, \mathbb{C}^{*}\right), \mathbb{T}_{H^{\prime}}=\operatorname{Hom}\left(H^{\prime}, \mathbb{C}^{*}\right), \mathbb{T}_{C}=\operatorname{Hom}\left(C, \mathbb{C}^{*}\right)$ и $\mathbb{T}_{G}=\mathbb{T}_{H} \times \mathbb{T}_{H^{\prime}} \times \mathbb{T}_{C}$. Группа $H$ гомоморфно отображается в $\mathbb{T}_{H^{\prime}}$ по формуле

$$
h \in H \mapsto\left\{h^{\prime} \mapsto \chi_{C}\left(\left\langle h, h^{\prime}\right\rangle\right)\right\}, \quad \chi_{C} \in \mathbb{T}_{C} .
$$

Обозначим через $H_{\chi}$ ядро этого отображения. Если $\chi \in \mathbb{T}_{H^{\prime}}$, то пусть $h(\chi)-$ сдвиг характера $\chi$ на образ элемента $h$ в $\mathbb{T}_{H^{\prime}}$. Имеем $h\left(\chi_{H^{\prime}}\right)(p)=\chi_{H^{\prime}}(p) \chi_{C}(\langle h, p\rangle)$ для всех $p \in H^{\prime}$. Для любого $\chi \in \mathbb{T}_{G}, \chi=\chi_{H} \otimes \chi_{H^{\prime}} \otimes \chi_{C}$, пусть $G_{\chi}$ - подмножество в $G$, определяемое условием

$$
g \in G_{\chi} \Longleftrightarrow \chi\left(g n g^{-1}\right)=\chi(n) \text { для всех } n \in H^{\prime} C .
$$

Тогда $G_{\chi}$ - нормальный делитель в $G$. Он зависит только от $\chi_{C}, G_{\chi}=H_{\chi} H^{\prime} C$, $H_{\chi}=G_{\chi} \cap H$, и $\chi \mid G_{\chi}$ является характером группы $G_{\chi}[7]$.

Определение 1. Пусть $V_{\chi}$ - пространство комплекснозначных функций $f$ на $G$, удовлетворяющих следующим условиям:

1) $f(g h)=\chi(h) f(g)$ для всех $h \in G_{\chi}$;

$2)$ носитель $\operatorname{Supp}(f)$ содержится в конечном числе левых классов смежности подгруппы $G_{\chi}$.

Левые сдвиги определяют представление $\pi_{\chi}$ группы $G$ в пространстве $V_{\chi}$.

Предложение 1. Справедливы следующие утверждения:

1) пространства $V_{\chi}$ не содержат нетривиальных подпространств, инвариантных относительно группы $G$;

2) линейные преобразования пространства $V_{\chi}$, перестановочные с операторами представления $\pi_{\chi}$, кратны тождественному преобразованию;

3) представления $V_{\chi}$ u $V_{\chi^{\prime}}$ эквивалентны тогда и только тогда, когда выполнены следующие условия:

(a) $\chi_{C}=\chi_{C}^{\prime}$,

(b) существует такое $h \in H$, ито $\chi_{H^{\prime}}^{\prime}=h\left(\chi_{H^{\prime}}\right)$,

(c) $\chi_{H}^{\prime}(h)=\chi_{H}(h)$ для всех $h \in H_{\chi}$ или, эквивалентно, существует такое $t \in \mathbb{T}_{H / H_{\chi}}=\operatorname{Hom}\left(H / H_{\chi}, \mathbb{C}^{*}\right)$, ито $\chi_{H}^{\prime}=t\left(\chi_{H}\right)$.

Здесь тор $\mathbb{T}_{H / H_{\chi}}$ действует сдвигами на объемлющем торе $\mathbb{T}_{H}$. Таким образом, классы эквивалентности представлений $V_{\chi}$ отвечают орбитам групп $\mathbb{T}_{H / H_{\chi}} \times H / H_{\chi}$ на всех подмножествах $\mathbb{T}_{H} \times \mathbb{T}_{H^{\prime}} \times\left\{\chi_{C}\right\}$ в торе $\mathbb{T}_{G}$.

*Автор поддержан грантами РФФИ 08-01-00095-а, ведущих научных школ 1987.2008.1, INTAS 05-1000008-8118. 
Группа $G$ является полупрямым произведением групп $H$ и $H^{\prime} C$, и доказательство утверждений 2 и 3 получается использованием формализма Макки ([4], [8]), описывающего категорию индуцированных представлений полупрямых произведений дискретных абелевых групп (см. [1, гл. 1], [9, гл. 1]). Будем говорить, что $\chi_{C}-$ характер общего положения, если форма $\chi_{C}\left(\left\langle h, h^{\prime}\right\rangle\right)$, $h \in H, h^{\prime} \in H^{\prime}$, невырожденна по обоим аргументам. Тогда $H_{\chi}=(0)$. Для доказательства утверждения 1 в случае характера $\chi_{C}$ общего положения нужно реализовать представление $\pi_{\chi}$ в пространстве регулярных функций на торе $\mathbb{T}_{H}$ и воспользоваться стандартным аргументом эргодичности (в топологии Зарисского). Для случая, когда $G_{\chi}$ - подгруппа конечного индекса, представление $\pi_{\chi}$ будет конечномерным. Общий случай сводится к этим двум.

Ограничение функций на группе $G$ на подгруппу $H$ определяет биекцию $V_{\chi} \stackrel{\sim}{\rightarrow} \mathscr{D}\left(H, H_{\chi}\right)$, где $\mathscr{D}\left(H, H_{\chi}\right)$ - пространство таких функций $f$ на $H$, что $f\left(h h_{1}\right)=\chi\left(h_{1}\right) f(h)$ для всех $h_{1} \in H_{\chi}$ и носитель $\operatorname{Supp}(f)$ содержится в конечном числе классов смежности подгруппы $H_{\chi}$. В пространстве $\mathscr{D}\left(H, H_{\chi}\right)$ имеется базис, состоящий из функций $\delta_{n}, n \in H / H_{\chi}$, сосредоточенных на классе смежности $n H_{\chi}$ подгруппы $H_{\chi}$. Такие функции, вообе говоря, нельзя выбрать однозначно, но если фиксировать множество $\bar{H} \subset H, 0 \in \bar{H}$, представителей классов смежности, то условие $\delta_{n}(\bar{n})=1$ (где $\bar{n}-$ представитель класса $n$ ) определяет базис однозначно. Тогда характер представления $\pi_{\chi}$ можно определить как след матриц операторов $\pi_{\chi}(g)$ в этом базисе. Легко видеть, что если подгруппа $G_{\chi}$ имеет бесконечный индекс в $G$, то операторы представления $\pi_{\chi}$ могут не иметь следа (соответствующая сумма диагональных элементов будет расходиться). Простейший пример: $G=\operatorname{Heis}(3, \mathbb{Z}), H=H^{\prime}=C=\mathbb{Z},\langle-,-\rangle-$ умножение в кольце $\mathbb{Z}$.

Чтобы определить, тем не менее, характер представления, нужно воспользоваться конструкцией, хорошо известной в теории групп петель $[6, \S 14.1]$, и добавить к группе $G$ «вращения петли». В нашей ситуации это означает, что группу $G$ нужно расширить до полупрямого произведения $\widehat{G}=G \rtimes A$, где $A \subset \operatorname{Hom}\left(H, H^{\prime}\right)$ - нетривиальная подгруппа.

Определение 2. Пусть $k \in A$ и зафиксирован элемент $r \in H$. Пусть

$$
k(m, p, c)=\left(m, p+k(m), c+\frac{1}{2}\langle m-r, k(m)\rangle\right), \quad \text { где } m \in H, p \in H^{\prime}, c \in C .
$$

Предложение 2. Выражение для $k$ определяет автоморфизм группы $G$, если выполнены следующие условия:

1) $\left\langle m, k\left(m^{\prime}\right)\right\rangle=\left\langle m^{\prime}, k(m)\right\rangle$ для всех $m, m^{\prime} \in H$;

2) $\langle m-r, k(m)\rangle \in 2 C$ для всех $m \in H$.

Предположим, кроме того, что

3) $k\left(H_{\chi}\right) \subset \operatorname{Ker}\left(\chi_{H^{\prime}}\right)$.

Представление группы $G$ в пространстве $V_{\chi}$ продолжается до представления $\hat{\pi}_{\chi}$ расширенной группы $\widehat{G}$ в том же пространстве. В базисе $\delta_{n}, n \in H / H_{\chi}$, операторы нового представления имеют вид

$$
\begin{aligned}
\hat{\pi}_{\chi}(m, p, c, k) \delta_{n}= & \chi_{C}(c) \chi_{H^{\prime}}(p+k(n-m)) \varrho(n, m) \\
& \times \chi_{C}\left(\langle n-m, p\rangle+\frac{1}{2}\langle n-m-r, k(n-m)\rangle\right) \delta_{n-m \bmod H_{\chi}},
\end{aligned}
$$


где числа $\varrho(n, m) \in \mathbb{C}^{*}$ могут зависеть от выбора $\bar{H}$, но $\varrho(n, m)=\chi_{H}(m)$, если $m \in H_{\chi}$. Пусть

$\left(\mathbb{T}_{C} \times A\right)_{+}:=\left\{(\chi, k) \in \mathbb{T}_{C} \times A:\left|\chi_{C}(\langle n, k(n)\rangle)\right|<1\right.$ для всех $\left.n \in H / H_{\chi}, n \neq 0\right\}$ - соответствие в $\mathbb{T}_{C} \times A$ и $A(\chi)$ - проекция на $A$ множества $\left(\mathbb{T}_{C} \times A\right)_{+} \cap$ $(\{\chi\} \times A)$.

Теорема 1. Если $\widehat{G}(\chi)=G \times A(\chi) \subset \widehat{G}, m o$

1) след $\operatorname{Tr} \hat{\pi}_{\chi}(g)$ существует для всех $g \in \widehat{G}(\chi)$;

2) если $g=(m, p, c, k), k \in A(\chi)$, mo $\operatorname{Tr} \hat{\pi}_{\chi}(g)=0$ для $m \notin H_{\chi}, a$ для $m \in H_{\chi}$ имеем

$$
\operatorname{Tr} \hat{\pi}_{\chi}(g)=\chi_{H}(m) \chi_{H^{\prime}}(p) \chi_{C}(c) \sum_{n \in H / H_{\chi}} \chi_{H^{\prime}}(k(n)) \chi_{C}\left(\langle n, p\rangle+\frac{1}{2}\langle n-r, k(n)\rangle\right) .
$$

Пример. Пусть $C=\mathbb{Z}, \chi_{C}(c)=\lambda^{c}, \lambda \in \mathbb{C}^{*}$. Тогда $A(\chi)=A_{+} \coprod A_{-}$, где $A_{ \pm}=\{k \in A:$ форма $\langle m, k(m)\rangle$ положительно (отрицательно) определена на $H / H_{\chi}$, если $\left.|\lambda| \lessgtr 1\right\}$.

Добавим еще одно условие на $k$ :

4) $\chi_{C}\left(\frac{1}{2}\langle m-r, k(m)\rangle\right)=1$ для всех $m \in H_{\chi}$.

В предыдущем примере это условие выполнено, если $\lambda$ - элемент бесконечного порядка в $\mathbb{C}^{*}$. Заметим, что величина из условия 4) всегда равна \pm 1 .

Пусть $\mathbb{T}_{A}=\operatorname{Hom}\left(A, \mathbb{C}^{*}\right)$ и $\mathbb{T}_{\widehat{G}}=\mathbb{T}_{G} \times \mathbb{T}_{A}$. Если $\hat{\chi}=\left(\chi, \chi_{A}\right) \in \mathbb{T}_{\widehat{G}}$, то положим $\hat{\pi}_{\hat{\chi}}=\hat{\pi}_{\chi} \otimes \chi_{A}$. Тогда $\operatorname{Tr} \hat{\pi}_{\hat{\chi}}=\operatorname{Tr} \hat{\pi}_{\chi} \cdot \chi_{A}$. Для данного $g \in \widehat{G}(\chi)$ след $\operatorname{Tr} \hat{\pi}_{\hat{\chi}}(g)$ определен как функция на области $T^{\prime}=\mathbb{T}_{H} \times \mathbb{T}_{H^{\prime}} \times \mathbb{T}_{C}(k) \times \mathbb{T}_{A}$ в торе $\mathbb{T}_{\widehat{G}}$, где $\mathbb{T}_{C}(k)$ - проекция на $\mathbb{T}_{C}$ множества $\left(\mathbb{T}_{C} \times A\right)_{+} \cap\left(\mathbb{T}_{C} \times\{k\}\right)$. Определим действие группы $\mathbb{T}_{H / H_{\chi}} \times H$ на множестве $\mathbb{T}_{H} \times \mathbb{T}_{H^{\prime}} \times\left\{\chi_{C}\right\} \times \mathbb{T}_{A} \subset T^{\prime}$ формулой

$$
(t, h)\left(\chi_{H}, \chi_{H^{\prime}}, \chi_{C}, \chi_{A}\right)=\left(t\left(\chi_{H}\right), h\left(\chi_{H^{\prime}}\right), \chi_{C}, \chi_{A}^{\prime}\right)
$$

где

$$
\chi_{A}^{\prime}(k)=\chi_{A}(k) \chi_{H^{\prime}}(k(h)) \chi_{C}\left(\frac{1}{2}\langle h-r, k(h)\rangle\right), \quad k \in A .
$$

Определим $\mathscr{M}_{G}(k), k \in A$, как факторпространство области $T^{\prime}$ по такому действию. Имеем диаграмму расслоений $\mathscr{M}_{G}(k) \rightarrow \mathscr{A}_{G}(k) \rightarrow \mathbb{T}_{C}(k)$, последнее со слоем $\mathbb{T}_{H^{\prime}} / \operatorname{Im} H$. Если $\overline{\mathscr{A}}_{G}(k)$ - универсальная накрывающая многообразия $\mathscr{A}_{G}(k)$, то положим $\overline{\mathscr{M}}_{G}(k)=\mathscr{M}_{G}(k) \times_{\mathscr{A}_{G}(k)} \overline{\mathscr{A}}_{G}(k)$. На накрытии $\varphi: \overline{\mathscr{M}}_{G}(k) \rightarrow$ $\mathscr{M}_{G}(k)$ действует дискретная группа $\Gamma=\pi_{1}\left(\mathscr{A}_{G}(k)\right)$.

Теорема 2. Справедливы следующие утверждения:

1) для данного $g=(m, p, c, k) \in \widehat{G} \operatorname{Tr} \hat{\pi}_{\hat{\chi}}(g)$ инвариантен относительно действия (4) и определяет голоморфную функиию $F_{g}=F_{g}(\hat{\chi})$ на $\mathscr{M}_{G}(k)$;

2) пусть $\hat{\chi}, \hat{\chi}^{\prime} \in \mathbb{T}_{\widehat{G}}$; представления $\hat{\pi}_{\hat{\chi}} u \hat{\pi}_{\hat{\chi}^{\prime}}$ эквивалентны тогда и только тогда, когда $\widehat{G}(\chi)=\widehat{G}\left(\chi^{\prime}\right)$ u $F_{g}(\hat{\chi})=F_{g}\left(\hat{\chi}^{\prime}\right)$ для всех $g \in \widehat{G}(\chi)$;

3) для данного $g=(m, p, c, k) \in \widehat{G}$ функиия $\varphi^{*} F_{g}$ является автоморфной формой на многообразии $\overline{\mathscr{M}}_{G}(k)$ относительно группь $\Gamma$.

Последнее утверждение аналогично известному свойству характеров представлений аффинных алгебр Каца-Муди ([3], [6]). 
Пример. Пусть $G=\operatorname{Heis}(3, \mathbb{Z}), A=\mathbb{Z}, r=1, \widehat{G}=G \rtimes \mathbb{Z}, \chi_{C}(c)=\lambda^{c}$, $\chi_{C} \in \mathbb{T}_{C}(k>0)=\{0<|\lambda|<1\}$. Тогда $\mathbb{T}_{H^{\prime}} / \operatorname{Im} H=: E_{\lambda}$ - эллиптическая кривая, где $\mathbb{T}_{H^{\prime}}=\mathbb{C}^{*}, \operatorname{Im} H=\left\{\lambda^{\mathbb{Z}}\right\}$. Имеем [2]

$$
\begin{gathered}
\operatorname{Pic}\left(E_{\lambda}\right)=H^{1}\left(E_{\lambda}, \mathscr{O}^{*}\right)=H^{1}\left(H, \mathscr{O}^{*}\left(\mathbb{T}_{H^{\prime}}\right)\right) \rightarrow \operatorname{Hom}\left(H, H^{\prime}\right)=A \ni k, \\
\operatorname{Pic}\left(E_{\lambda}\right)=\left\{\varphi(n, z)=a^{-n} z^{-k n} \lambda^{-\frac{1}{2} k n(n-1)}: a \in \mathbb{C}^{*}, k \in \mathbb{Z}, z \in \mathbb{T}_{H^{\prime}}\right\} .
\end{gathered}
$$

Пусть линейное расслоение $L$ отвечает коциклу $\varphi$. Тогда

$$
H^{0}\left(E_{\lambda}, L\right)=\left\{f(z), z \in \mathbb{T}_{H^{\prime}}: f\left(\lambda^{n} z\right)=\varphi(n, z) f(z)\right\}
$$

и достаточно, чтобы $f(\lambda z)=a^{-1} z^{-k} f(z)$. Тета-ряды

$$
\vartheta_{p, k, a}(z, \lambda):=z^{p} \sum_{n \in \mathbb{Z}} a^{n} z^{k n} \lambda^{n p+\frac{1}{2} k n(n-1)}
$$

(это ряды Пуанкаре относительно $\varphi$ ) сходятся для всех $z \in \mathbb{C}^{*}, 0<|\lambda|<1$, $k>0$ и образуют для $0 \leqslant p<k$ базис в пространстве $H^{0}\left(E_{\lambda}, L\right)$. Наконец,

$$
\operatorname{Tr} \hat{\pi}_{\hat{\chi}}(0, p, c, k)=\lambda^{c} t^{k} \vartheta_{p, k, 1}(z, \lambda), \quad(z, \lambda) \in \mathscr{A}_{G}(k), t \in \mathbb{T}_{A} .
$$

В этой ситуации утверждение 3 ) теоремы 2 очевидно (тета-функции, поднятые на $\overline{\mathscr{A}}_{G}(k)=\mathbb{C} \times\{$ верхняя полуплоскость $\}$, являются якобиевыми модулярными формами относительно действия подгруппы группы $(\mathbb{Z} \oplus \mathbb{Z}) \rtimes \mathrm{SL}(2, \mathbb{Z}))$. Утверждение 2) следует в одну сторону из прямого вычисления, а в обратную сторону из теоремы о вложении эллиптической кривой в проективную плоскость с помощью тета-функций.

В более общей ситуации, когда $H, H^{\prime}-$ группы без кручения, $C=\mathbb{Z}$, $k: H \rightarrow H^{\prime}-$ мономорфизм с конечным коядром, $A=\mathbb{Z} k$ и форма $\langle-, k(-)\rangle$ положительно определена, мы имеем два двойственных абелевых многообразия, $E=\mathbb{T}_{H^{\prime}} / \operatorname{Im} H$ и $E^{\prime}=\mathbb{T}_{H} / \operatorname{Im} H^{\prime}$. Расслоение Пуанкаре $\mathscr{P}$ над $E \times E^{\prime}$ определяется как факторпространство тора $\mathbb{T}_{H} \times \mathbb{T}_{H^{\prime}} \times \mathbb{C}^{*}$ по действию группы $H \times H^{\prime},\left(h, h^{\prime}\right)\left(\chi_{H}, \chi_{H^{\prime}}, u\right)=\left(h^{\prime}\left(\chi_{H}\right), h\left(\chi_{H^{\prime}}\right), u \chi_{H}(h) \chi_{H}\left(h^{\prime}\right) \chi_{C}\left(\left\langle h, h^{\prime}\right\rangle\right)\right), h \in H$, $h^{\prime} \in H^{\prime}, u \in \mathbb{C}^{*}$. Морфизм $k$ определяет изогению $\varphi_{k}: E \rightarrow E^{\prime}$. Имеем точную последовательность

$$
1 \rightarrow \mathbb{T}_{H^{\prime} / H} \rightarrow \operatorname{Ker} \varphi_{k} \rightarrow H^{\prime} / H \rightarrow 1
$$

и, согласно [5], конечную группу Гейзенберга $\widetilde{\operatorname{Ker}} \varphi_{k}$, являющуюся центральным расширением группы Ker $\varphi_{k}$. Выбор инвариантной формы старшей степени на торе $\mathbb{T}_{H^{\prime}} \times \mathbb{T}_{C} \times \mathbb{T}_{A}$ определяет интегрирование голоморфных функций на $\mathscr{M}_{G}(k)$ по некоторой мере $d \mu$.

Теорема 3. Пусть $L:=\left(\operatorname{Id} \times \varphi_{k}\right)^{*} \mathscr{P}$. Тогда справедливы следующие утверждения:

1) для всех $g=(m, p, c, k) \in \widehat{G}$ значения характеров $\operatorname{Tr} \hat{\pi}_{\hat{\chi}}(g) \chi_{C}^{-1}(c) \chi_{A}^{-1}(k)$ являются тета-функииями для расслоения $L$;

2) если $\hat{\chi}=1 \otimes \chi_{H^{\prime}} \otimes \chi_{C} \otimes 1$, то функиии $\operatorname{Tr} \hat{\pi}_{\hat{\chi}}(0, p, 0, k)$ для $p \in H^{\prime} \bmod k(H)$ образуют базис в пространстве $H^{0}(E, L)$; он является стандартным базисом Мамфорда [5] для действия группы $\widetilde{\operatorname{Ker}} \varphi_{k}$ на пространстве $H^{0}(E, L)$; 
3) если $g=(0, p, c, k), g^{\prime}=\left(0, p^{\prime}, c^{\prime}, k^{\prime}\right)$ не сопряюсены в $\widehat{G}$, то

$$
\int_{\mathscr{M}_{G}(k)} \operatorname{Tr} \hat{\pi}_{\hat{\chi}}(g) \overline{\operatorname{Tr} \hat{\pi}_{\hat{\chi}}\left(g^{\prime}\right)} d \mu=0 .
$$

В условиях теоремы 3 можно получить утверждение 2 теоремы 2 , используя теорему Лефшеца о проективном вложении абелевых многообразий с помощью тета-функций.

Исходный характер $\left.\chi_{H}\right|_{H_{\chi}} \otimes \chi_{H^{\prime}} \otimes \chi_{C}$ можно восстановить, зная $\operatorname{Tr} \hat{\pi}_{\chi}(g)$.

Предложение 3. $\chi_{H}(m) \chi_{H^{\prime}}(p) \chi_{C}(c)=\lim _{l \rightarrow+\infty} \operatorname{Tr} \hat{\pi}_{\chi}(m, p, c, l k)$, если $m \in$ $H_{\chi}, k \in A(\chi), l \in \mathbb{N}$.

Граница $\partial \mathbb{T}_{C}(k)$ области $\mathbb{T}_{C}(k)$ может содержать такие характеры $\chi \in \mathbb{T}_{C}$, что $H_{\chi}$ имеет конечный индекс в $H$. Значения этих характеров имеют конечный порядок в группе $\mathbb{C}^{*}$. Если группа $H_{\chi}$ тривиальна, то пространство $V_{\chi}$ не зависит от $\chi$.

Предложение 4. Пусть $\chi_{C} \in \mathbb{T}_{C}(k)$ u груnпа $H_{\chi}, \chi=\chi_{H} \otimes \chi_{H^{\prime}} \otimes \chi_{C}$, mpuвиальна. Если $\chi^{\prime} \in \partial \mathbb{T}_{C}(k)$ - характер конечного порядка, то пространство $V_{\chi}$ представления $\pi_{\chi}$ разлагается в бесконечную прямую сумму конечномерных представлений вида $V_{\mu}$, где $\mu=\mu^{\prime} \otimes \chi_{H^{\prime}} \otimes \chi^{\prime}$ и $\mu^{\prime}$ пробегает все характерь грynnъı $H_{\chi^{\prime}}$.

Автор глубоко признателен М. Бургеру и А. М. Вершику за полезные обсуждения.

\section{ЛИТЕРАТУРА}

[1] И. Н. Бернштейн, А. В. Зелевинский, УМН, 31:3 (1976), 5-70. [2] L. Gerritzen, Math. Ann., 196 (1972), 323-346. [3] V. Kac, D. Peterson, Adv. Math., 53:2 (1984), 125-264. [4] G. Mackey, Amer. J. Math., 73 (1951), 576-592. [5] D. Mumford, Invent. Math., 1 (1966), 287-354. [6] Э. Прессли, Г. Сигал, Групnъ петель, Мир, М., 1990. [7] T. Pytlik, Monatsh. Math., 93:4 (1982), 309-328. [8] Ж.-П. Серр, Линейные представления конечных групn, Мир, М., 1970. [9] M.-F. Vignéras, Représentations $l$-modulaires d'un groupe réductif $p$-adique avec $l \neq p$, Birkhäuser, Boston, MA, 1996.

Математический институт им. В. А. Стеклова РАН e-mail: parshin@mi.ras.ru

Поступило в редакцию 28 декабря 2009 г.

Заведующая редакцией и научный редактор Г. М. Цукерман

Сдано в набор 26.03.2010. Подписано к печати 26.04.2010. Формат 70×100/16 Печать офсетная. Усл. печ. л. 7,8. Усл. кр.-отт. 2,0 тыс. Бум. л. 3,0 Уч.-изд. л. 8,0. Тираж 247 экз. Заказ 197.

Учредитель: Российская академия наук

Адрес редакции: 117966 Москва, ГСП-1, ул. Губкина 8, комн. 624. Тел. 938-37-56

Издатель: Российская академия наук, Издательство «Наука», 117997 Москва, Профсоюзная, ул. 90

Отпечатано в ППП «Типография «Наука», 121099 Москва, Шубинский пер., 6 